\title{
Origami-like creases in sheet materials for compliant mechanism design
}

\author{
K. C. Francis, J. E. Blanch, S. P. Magleby, and L. L. Howell \\ Department of Mechanical Engineering, Brigham Young University, Provo UT, 84602, USA
}

Correspondence to: L. L. Howell (lhowell@byu.edu)

Received: 19 April 2013 - Accepted: 1 July 2013 - Published: 15 November 2013

\begin{abstract}
The purpose of this research is to evaluate the creasing of non-paper sheet materials, such as plastics and metals, to facilitate origami-based compliant mechanism design. Although it is anticipated that most origami-based design will result from surrogate folds (indirect methods of replacing the crease), it is valuable to provide information that may help in more direct approaches for origami-based design in materials other than paper. Planar sheets of homogeneous material are considered as they maintain the principles fundamental to origami (flat initial state, low cost, readily available). The reduced stiffness along the axis of the crease is an enabling characteristic of origami. Hence a metric based on the deformation of the crease compared to the deformation of the panels enables engineering materials to be evaluated based on their ability to achieve the "hinge-like" behavior observed in folded paper. Advantages of both high and low values of this metric are given. Testing results (hinge indexes, residual angles, localized hinge behavior and cyclic creasing to failure) are presented for various metals and polymers. This methodology and subsequent findings are provided to enable origami-based design of compliant mechanisms.
\end{abstract}

\section{Introduction}

Folded materials provide an opportunity for inspiration in mechanism design. Origami, the ancient art of paper folding, represents a unique way of creating mechanisms (Dureisseix, 2012). For centuries origami artists have designed in the highly available medium of paper, producing a variety of folding patterns ranging from statuesque representations of physical phenomena to animated creatures capable of motion. Some designs have the ability to be tessellated, enabling surprising expansion and contraction of the structure. The past few decades have brought a heightened interest in origami which has resulted in the increased interest in developing science, technology and computational methods that could be used to solve modern complex problems.

Origami can be viewed as a compliant mechanism (Greenberg et al., 2011; Dureisseix, 2012) as it achieves its motion from the deflection of flexible members (Howell, 2001). Origami may also be considered to fit into the class of lamina emergent mechanisms (Jacobsen et al., 2009, 2010), due to the planar nature of the paper (lamina) and the configura- tion emerging from the plane of fabrication. Designs ideated through folding are quite different from those synthesized through traditional engineering methods and present desirable characteristics as they are made from a single sheet, employ only one manufacturing process (folding) and are compact. These benefits solicit the transfer of folding designs into materials which are able to meet a broader set of design constraints (e.g. temperature, humidity, biocompatibility, strength, stiffness, combustion, etc.).

There are many ways to achieve crease-like behavior. Creating a "surrogate-crease" has been accomplished by perforation, thinning, a variety of through cuts leaving an optimized geometry which functions similar to a joint (Ferrell et al., 2011; Jacobsen et al., 2009; Wilding et al., 2012) and mechanical hinges. While these methods are well studied and the results provide the desired revolute motion, the intent of this paper is to characterize the behavior of a material when it has been mechanically creased through a folding process. 


\section{Background}

Materials that have origami-like creases have the ability to both fold and unfold. Hence a material that tends to bend at a previous fold is a material that has origami-like creases. Some materials do not crease (e.g. sheet metal) because they do not exhibit decreased stiffness along the fold. Creases develop when the bending stress is greater than the elastic limit of the material (Gagliardi and Gruntfest, 1950). The formation of a crease resets the elastic memory to a non-zero angle; the harder the crease is pressed, the greater the residual angle (Demaine et al., 2011).

Various levels of crease characterization exist amongst sheet materials. Paper is a well understood material in the context of creasing. Textiles have become better understood through study into creaseproofing and pleating. New manufacturing methods and a recent interest in folded designs of various size scales and materials warrant better characterization of creases in materials beyond paper and textiles. Work in paper and textile folding and the related manufacturing methods are discussed in the following sections. Building on this knowledge helps to characterize the crease properties of non-paper sheet materials, especially polymers and metals, which can expand the possibilities of origami-inspired design.

\subsection{Paper}

In the realm of creasing, the properties of paper and paper derivatives are among the most understood. Paper behavior is often simplified by considering it unstretchable. Overall dimensions of the sheet are maintained when paper is creased, making it applicable, or mappable back to a plane (Kergosien et al., 1994). Rigid-foldable origami is able to be constructed without stretching, deforming or twisting its panels (Tachi, 2011; Wu and You, 2010). Folding does not allow tearing or self-penetration of the sheet (Demaine and O'Rourke, 2007).

In applications where paper has appreciable thickness, such as paperboard, additional characterization is found. Kinematically, creases act like a torsional spring, storing energy as the panels rotate relative to each other along the axis of the crease (Gardiner, 2011). The torsional spring, or equivalent joint as Dai (2008) used in their carton folding model, exhibits variable stiffness. This stiffness is dependent on the angular displacement experienced creating the fold. Primary and secondary foldings exhibit different stiffness properties due to the carton's delamination. Paperboard and corrugates often are precreased through mechanical die impacts. Experimental (Nagasawa et al., 2003) and finite element (Beex and Peerlings, 2009) models of penetration depth and crease performance have been explored.

\subsection{Textiles}

The stranded, woven nature of textiles enables them to withstand the effects of repeated folding much better than fibrous paper (Gardiner, 2011). Creaseproofing (Hearle et al., 1969; Gagliardi and Gruntfest, 1950) and pleating (Marsh, 1962; Morton and Hearle, 1962; Vigo, 1994) are well-developed, complementary specialties based on the science of creasing fabrics. Macro and micro material considerations lead to the crease properties of a specific fabric. Temperature, pressure, humidity and treatments also influence the formation of creases (Wingate and Mohler, 1984; Gardiner, 2011; Dureisseix, 2012).

\subsubsection{Manufacturing}

Advances in folding automation make folding a prime manufacturing method for sheet materials. Historically, deformable manipulation (or the manipulation of non-rigid materials) has been viewed as challenging as it must accommodate for deformations due to weight, dynamic forces and gripping forces (Karakerezis et al., 1994). However manipulating these non-rigid materials has been effective in the production of fast food containers, paper bags, clothing, and crash safety air bags (Balkcom and Mason, 2008). Flexibility and short setup times are found in incremental sheet forming operations as it is a CNC process (Allwood et al., 2005).

The automation of pleating is much like folding. Direct and reverse folds are created in pliable materials by a continuous machine for applications such as apparel, novelties, and filtration devices (Hochfeld, 1959). Increased ability to automate complex folding operations is seen in the continuous production of foldcores or modular isometric origami (Klett and Drechsler, 2011) in a variety of materials such as aluminum, stainless steel, copper, paper, composites and plastics.

Advantages of folding over traditional sheet forming methods center on maintaining a constant sheet thickness (minimizing profile variations) and lower forming energy requirements (Fischer et al., 2009; Elsayed and Basily, 2004).

\subsubsection{Folding in other materials}

Recent work has taken folding to a variety of size scales and materials. At the nano-level, graphene sheets, one atom thick, have been folded into stable configurations (Canford et al., 2009). Protein and DNA folding are widely explored (Rothemund, 2006; Demaine and O'Rourke, 2007). Microassembly of origami structures has been achieved through bidirectional folds with pre-stressed layers of actuation material (Bassik et al., 2009). Polymers folding through incorparated actuation has been realized (Ryu et al., 2012; Liu et al., 2012) and elastomeric folding has been evaluated (Martinez et al., 2012). The folding of plastics is used in product design to provide more rigid products with varying enclosed volume 


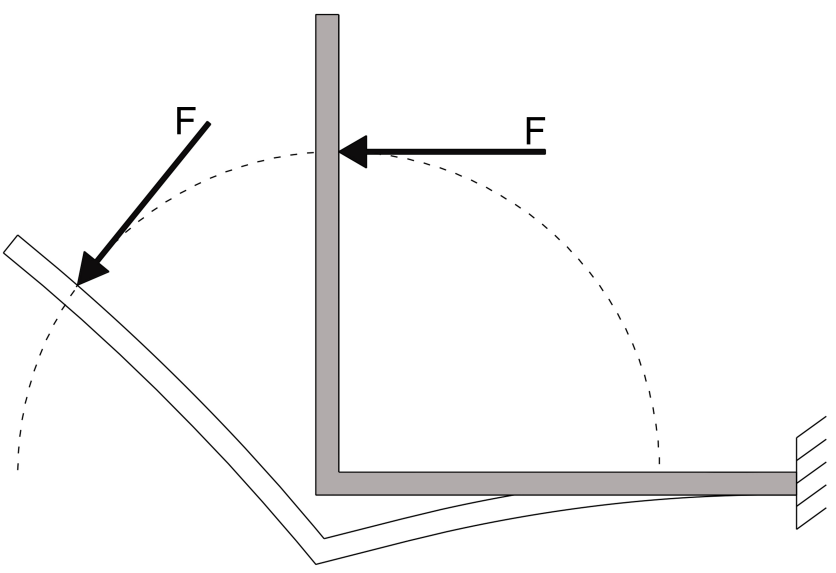

Figure 1. The base case: a creased sheet of material set to a 90 degree angle and cantilevered. A displacement load is applied to the initial state (shaded), causing it to deflect to the final state (not shaded). The displacement follows an arc centered at the initial location of the crease.

(e.g. expanding organization folders, Swab, 1940, collapsible kayaks, Willis, 2012, foldable plastic bowls, Fite et al., 2008).

The characteristics of folded materials are relatively unexplored outside of paper and textiles. The behavior of the crease in various engineering materials could make them more or less advantageous for various applications. A further understanding of folded planar material would enable designers to make better decisions in the development of their products.

Although it is anticipated that the majority of future origami-inspired designs will employ surrogate creases, there is also value in evaluating the possibility of creasing materials to more directly achieve origami-like behavior. The purpose of this paper is to better characterize the creasing of various engineering materials. This is done through (1) establishing a base case to facilitate evaluation and comparison, (2) developing crease performance metrics, (3) experimental testing of a range of polymers, metals and fiber based sheet goods.

\section{Approach}

The behavior of creases in sheet materials can be evaluated using a simple representation of creasing that translates well into the folding domain. Through keeping the case basic, findings can be extracted and transposed to guide designers in material selection and design considerations for folded designs.

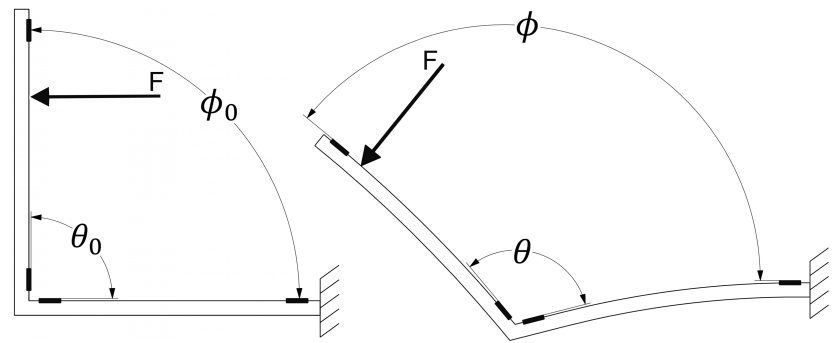

Figure 2. The image on the left represents the initial or unstrained state of the sample as viewed from above, and the right is an outward deflection of the sample. $\theta$ and $\phi$ are used to calculate the hinge index.

\subsection{Base case}

The base case is a cantilevered sheet of material that protrudes out from a fixed end condition, as illustrated in Fig. 1. At half of its length, the sheet is creased, creating two panels of equal size. The equilibrium, or low-energy, orientation of the panels is set to 90 degrees. Incremental displacements are applied to this base configuration, and panel deflections are observed.

The cantilevered approach was used to simulate an origami folding procedure. As origami does not have rigid constraints on the panels surrounding the crease, the ability of both panels to deflect is desired.

\subsection{Hinge parameter}

Characterizing crease behavior is beneficial to designers because of strain energy and rigid-foldable considerations in design. A metric for characterizing the folding behavior of various materials can be useful in evaluating and comparing performance. We propose a metric called the hinge parameter

$h=\frac{\theta-\theta_{0}}{\phi-\phi_{0}}$

where $\theta$ is the measure of the interior angle, or the angle of the tangents to the panels near the hinge, and $\phi$ is the measure of the exterior angle, or the angle of the tangents to the panels far from the hinge as illustrated in Fig. 2. Theoretical values of the hinge parameter range between 1 (a frictionless mechanical hinge) and 0 (a non-functioning hinge with motion from panel bending).

The behavior of creases in sheet materials can be evaluated using a simple representation of creasing that translates well into the folding domain. Through keeping the case basic, findings can be extracted and transposed to guide designers in material selection and design considerations for folded designs. 


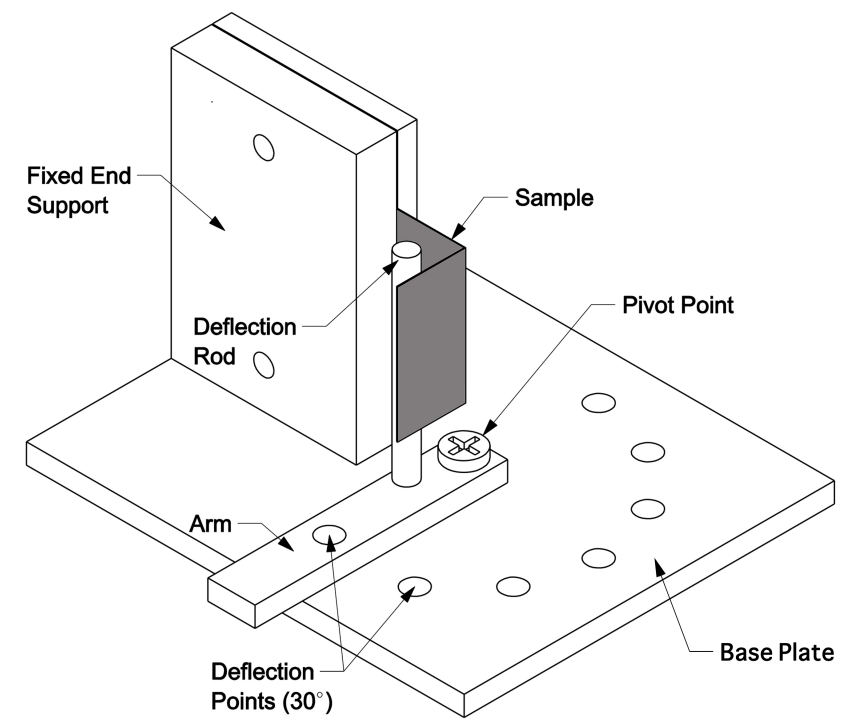

Figure 3. Isometric view of the test fixture. The clamped sample is shaded.

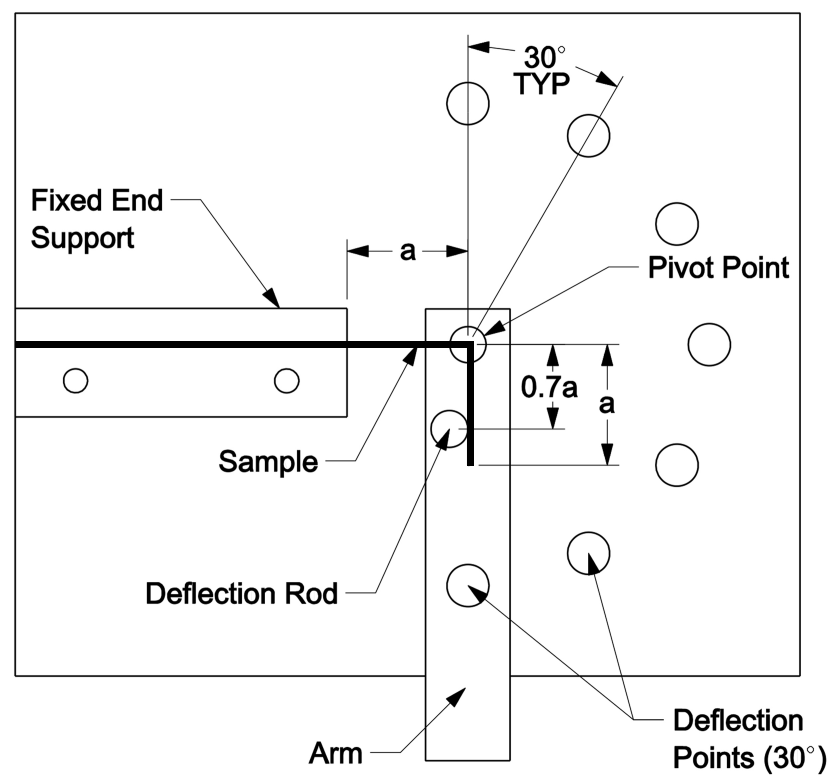

Figure 4. Top view of the test fixture. The sample can be seen in the center, clasped in the fixed end support.

\section{Experimental set-up}

A fixture for displacing material samples was designed and fabricated to perform the crease evaluation test uniformly, as shown in Figs. 3 and 4. The fixture consists of a base plate, a pivot arm with a deflection rod and a fixed-end support. On the rectangular baseplate, there are 7 locator positioning holes (labeled deflection points in Figs. 3 and 4 bored at 30 degree increments to form a semicircle. One end of a pivot arm is pinned about the center of the semicircle with the free

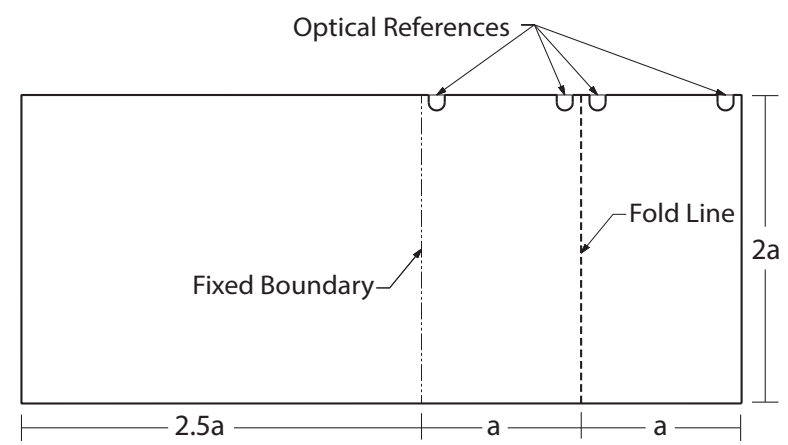

Figure 5. A view of the sample before creasing, with optical measurement locators and orientation marks shown. If the material has a rolling direction, the rolling direction would be in the horizontal axis in this figure.

end extending beyond the radius of the semicircle. This arm rotates in 30 degree steps through the semicircle and is held in place with a pin at any of the 7 locator holes. A deflection rod extendeds out of the arm and applies the displacement to the sample. This rod is positioned a radial distance of $a / \sqrt{2}$ from the pivot and offset from the arm's centerline $D / 2$ where $D$ is the diameter of the deflection rod.

\subsection{Sample preparation}

Samples of each material were sheared to the dimensions of $4.5 a \times 2 a$ (experimental data found later in this paper is based on a value of $a=2 \mathrm{~cm}$ and $D=0.635 \mathrm{~cm}$ ). In materials that exhibit anisotropic properties due to a clear rolling direction (which is common for sheet goods), the material was sheared with the longest dimension aligned with the rolling direction. The $4.5 a$ length is composed of three sections: a $2.5 a$ section which is clamped in the fixture to produce the fixed (cantilevered), end condition and a $2 a$ section which is bisected by a crease to create two panels with the crease centered between panels. Each sample was labeled (with material and iteration identifiers) and marked (with construction, orientation and measurement references) as shown in Fig. 5. Locations for optical reference features were offset from the crease by $0.1 \mathrm{~cm}$.

The samples were folded and creased in the following manner to minimize variance: fiber based materials were completely folded over by taking the protruding end and doubling it back such that it was flush with the clamping alignment mark. A bone folder was then used to impart a crease on this fold. The fold was then manipulated back to a 90 degree base angle. This same methodology was used for the polymers tested. Due to the forces required to work with metal sheets, a standard sheet metal bending hand brake was used to bend the sheets to a 90 degree angle. 


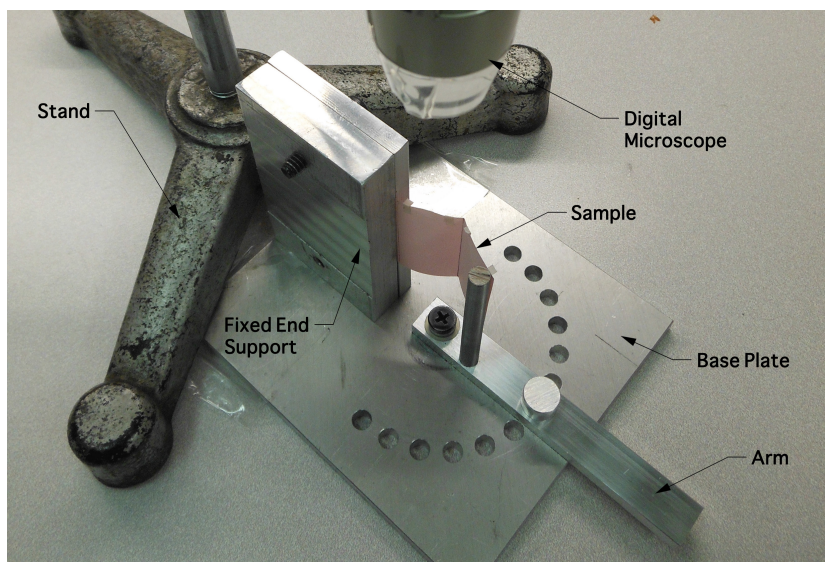

Figure 6. The test fixture with sample deflected to positive 90 degrees. Data is collected from the image captured by the Dino-Lite ${ }^{\mathrm{TM}}$ digital microscope which views the fixture from above.

\subsection{Hardware}

The sample was clamped by a fixed end support that rises vertically out of the base plate and is located opposite of the semicircle, a distance $a$ from the center pivot point. The fixed end support consists of two plates which clamp the sample material as the bolts connecting the plates are tightened. The samples were placed in the test fixture, as shown in Fig. 6.

Angle measurements were taken of the sample material at each 30 degree displacement position by placing a DinoLite $^{\mathrm{TM}}$ AM3011 digital microscope above the sample, viewing the sample's top edge. Digital images for each displacement position were processed in SolidWorks. Optical references served as the reference points for construction lines and subsequent angle measurements.

The experiments were performed in a controlled environment with temperature and relative humidity data logged. The averages and standard deviations for these are $20.1{ }^{\circ} \mathrm{C}$ $\left(0.59^{\circ} \mathrm{C}\right)$ and $13.4 \%(1.96 \%)$, respectively. The dimensions of the samples are $4 \times 9 \mathrm{~cm}(a=2 \mathrm{~cm})$.

\section{Results}

\subsection{Hinge index}

This work characterizes how well origami-like creases act as hinges in various materials, as characterized by the hinge index (Fig. 2 and Eq. 1). When materials were creased just once, the displacement of the panels, or flexing of the panels, was often greater than the angular rotation at the crease. Such behavior is manifest in a low hinge index. A traditional hinge (two rigid panels connected by a rotating pin) would have a hinge index of 1.

Table 1 lists the measured hinge index at three different angular deflections for 13 materials (4 metals, 6 polymers, Tyvek $^{\circledR}$ and 2 papers) of the same thickness $(0.25 \mathrm{~mm})$.
Table 1. Hinge Indexes and Residual Angles of $0.25 \mathrm{~mm}$ (0.01 in) Thick Materials.

\begin{tabular}{lcccc}
\hline Material & $30^{\circ}$ & $60^{\circ}$ & $90^{\circ}$ & $\begin{array}{c}\text { Residual } \\
\text { Angle }^{\circ}\end{array}$ \\
\hline Low Carbon Steel (1010) & 0.17 & 0.11 & 0.14 & 7.6 \\
Stainless Steel (304) & 0.22 & 0.13 & 0.11 & 9.4 \\
Aluminum (1100-H14) & 0.16 & 0.15 & 0.17 & 6.4 \\
Brass (Alloy 260 Half Hard) & 0.15 & 0.16 & 0.10 & 7.4 \\
\hline Polycarbonate & 0.12 & 0.12 & 0.10 & 1.6 \\
IR Plastic & 0.26 & 0.26 & 0.26 & 13.9 \\
Nylon & 0.08 & 0.08 & 0.15 & 7.4 \\
Acetal & 0.20 & 0.20 & 0.16 & 4.0 \\
PTFE & 0.37 & 0.16 & 0.12 & 1.8 \\
Polypropylene & 0.41 & 0.37 & 0.29 & 10.8 \\
\hline Tyvek ${ }^{\circledR}$ (1085D) & 0.64 & 0.49 & 0.44 & 0.1 \\
\hline Hard Fiber & 0.59 & 0.52 & 0.52 & 11.9 \\
Cardstock & 0.85 & 0.71 & 0.63 & 13.7 \\
\hline
\end{tabular}

Table 2. Hinge indexes and residual angles of other materials.

\begin{tabular}{lcccc}
\hline Material & $\begin{array}{c}\text { Thickness, } \\
\mathrm{mm}(\mathrm{in})\end{array}$ & $30^{\circ}$ & $60^{\circ}$ & $90^{\circ}$ \\
\hline Stainless Steel (304) & $0.05(0.002)$ & 0.20 & 0.14 & 0.09 \\
Aluminum (1100-H14) & $0.05(0.002)$ & 0.09 & 0.05 & 0.02 \\
Metallic Glass & $0.02(0.0009)$ & 0.08 & 0.05 & 0.09 \\
\hline PTFE & $0.13(0.005)$ & 0.03 & 0.00 & 0.01 \\
UHMW Polyethylene & $0.13(0.005)$ & 0.25 & 0.19 & 0.17 \\
UHMW Polyethylene & $0.51(0.020)$ & 0.44 & 0.28 & 0.20 \\
\hline Bee Paper Watercolor & $0.36(0.014)$ & 0.68 & 0.58 & 0.54 \\
Bee Paper Watercolor & $0.51(0.020)$ & 0.90 & 0.83 & 0.82 \\
Paper (AITOH OG1) & $0.05(0.002)$ & 0.34 & 0.27 & 0.26 \\
\hline
\end{tabular}

Paper products, or fiber-based materials, serve as a benchmark material for folding. The paper samples tested had the highest hinge indexes $(0.52$ to 0.85$)$ and the sheet metals tested gave lower hinge indexes $(0.10$ to 0.22$)$. Polymers exhibited a wide range of performance (0.08 to 0.41$)$. Tyvek $^{\circledR}$ spunbonded olefin, a product made of polymer fibers, exhibits a hinge index ( 0.44 to 0.64$)$ between that of the best polymer and that of the papers. Of the polymer sheets tested, polypropylene gave the highest hinge index values (0.29 to 0.41). Note that the hinge indexes are taken from positive deflections of the panels from their initial 90 degree state, where $\theta$ is a positive displacement (see Fig. 7). Negative angle displacements were also measured and show similar trends as those of the positive displacement angles.

Table 2 lists hinge indexes measured for other materials of interest, including metallic glass and origami paper. Unlike Table 1 where each entry has the same thickness, Table 2 has varying thickness because of the availabilities of stock material and so the results are not meant for direct comparison. 


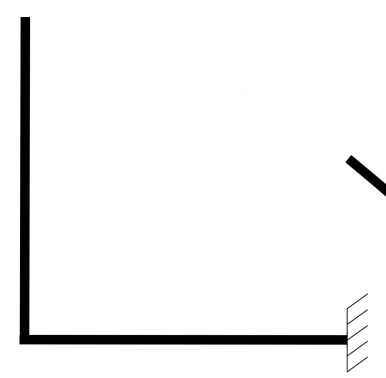

Initial Position

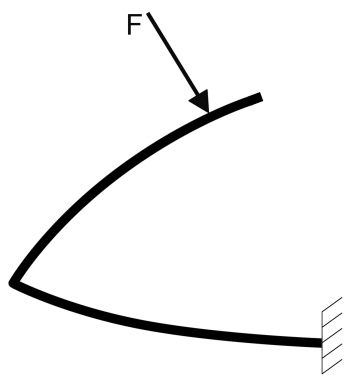

Negative Angles

Figure 7. Deflection angle orientations.

Table 3. Variation in hinge indexes and residual angles for different thicknesses of polypropylene.

\begin{tabular}{cccc|c}
\hline Thickness, mm (in) & $30^{\circ}$ & $60^{\circ}$ & $90^{\circ}$ & $\begin{array}{c}\text { Residual } \\
\text { Angle }^{\circ}\end{array}$ \\
\hline $0.25(0.010)$ & 0.41 & 0.37 & 0.29 & 10.8 \\
$0.43(0.017)$ & 0.39 & 0.39 & 0.31 & 12.2 \\
$0.51(0.020)$ & 0.49 & 0.56 & 0.56 & 23.1 \\
$1.02(0.040)$ & 0.60 & 0.60 & 0.60 & 28.5 \\
\hline
\end{tabular}

\subsection{Residual angle}

The residual angle is the angle that the sample returns to after being deflected 90 degrees from the original folded position. It is measured through changes in $\phi$, the angle measured further from the crease. Materials with low residual angles better resist permanent deformation from forces and loads, returning to the previous configuration when disturbances are removed. Thus they act like stiffer springs and require a greater input force to attain the same displacement. It is generally seen in fibers and polymers that greater residual angles correspond to higher hinge indexes, as shown in Table 1 . Tyvek ${ }^{\circledR}$ shows an opposite trend as it has a high hinge index and a low residual angle.

Design concepts of this are seen in Fig. 8, where the lowest picture shows that low hinge index materials (such as polycarbonate) return to their initial configuration while high hinge index materials (such as fibrous paper) do not. This concept could be leveraged in product development where materials with different hinge indexes would be used in different applications depending on how critical high restoration to the original configuration is.

\subsection{Thickness study}

The stiffness of the creased hinge increases as the material thickness increases, but the panel stiffness also increases with increasing thickness. The overall hinge index was higher for thicker sheets of the same material.
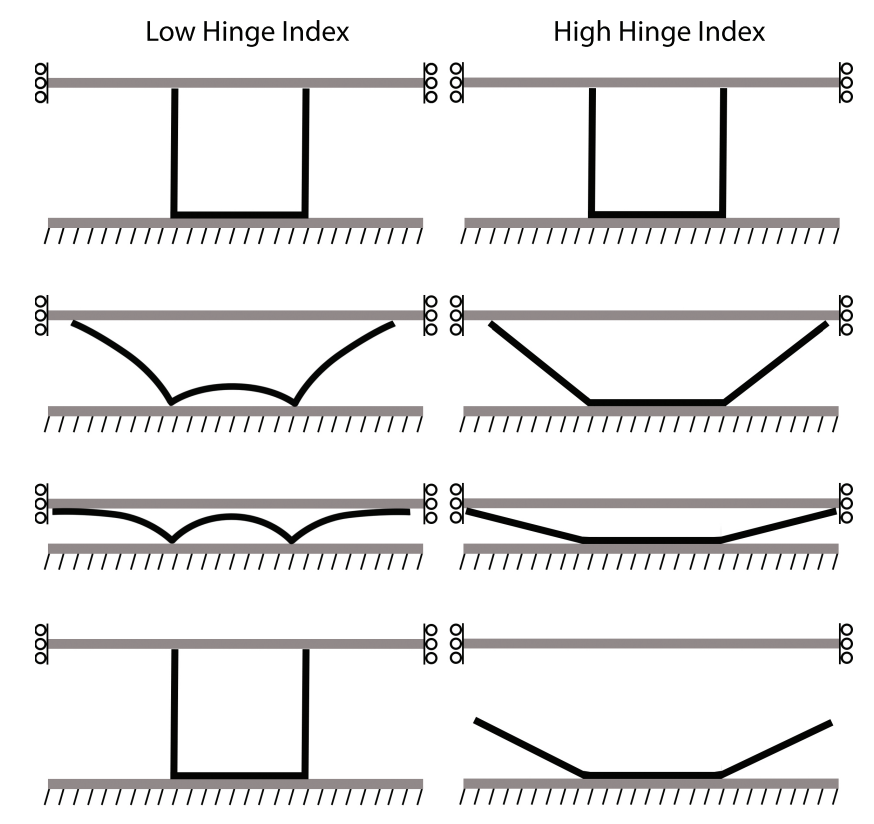

Figure 8. The schematic shown is based on photographs of polycarbonate (left) and fibrous paper (right) samples placed in a vice and compressed. Unlike previous samples, these samples each have a "U" shape that is formed by two 90 degree creases. Left side: low hinge index indicating panel warping, right side: fibrous paper or high hinge index indicating crease acting as a hinge.

A variety of samples were tested, varying the thickness and material properties. Table 3 illustrates how thickness impacts the fold characteristics of polypropylene. Once again the residual angle increases with an increasing hinge index. Table 4 reinforces the trend of a greater thickness leading to an increase in the hinge index, but with Tyvek ${ }^{\circledR}$ the residual angle decreases as the hinge index increases.

\subsection{Cyclic creasing}

The effects of cyclic creasing were explored. Creases in each material were manually cycled at the original crease location. Hinge index values were found for 1, 5, 10, 20, and 


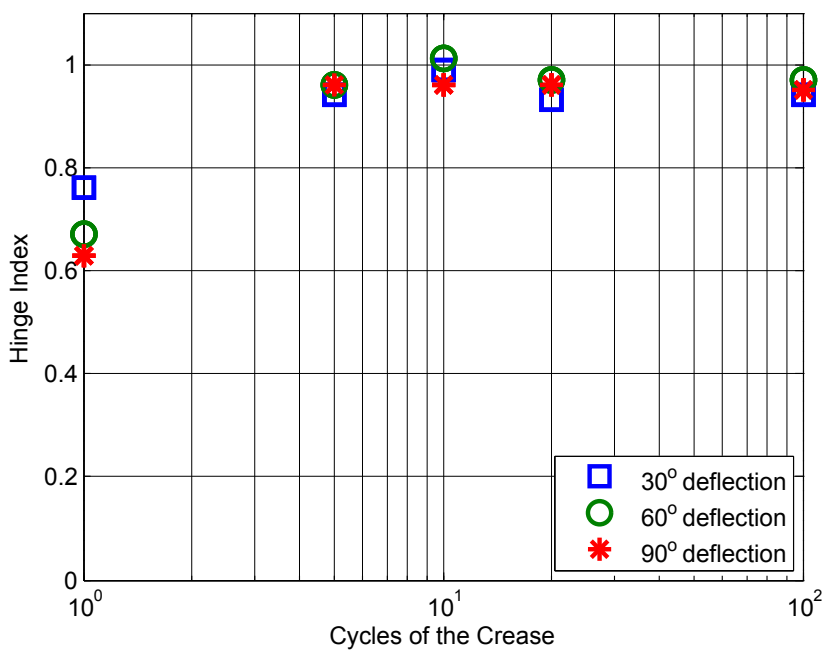

Figure 9. Cardstock ( $0.25 \mathrm{~mm}$ thick): hinge index verses cycles of the crease. A strong asymptotic behavior is seen.

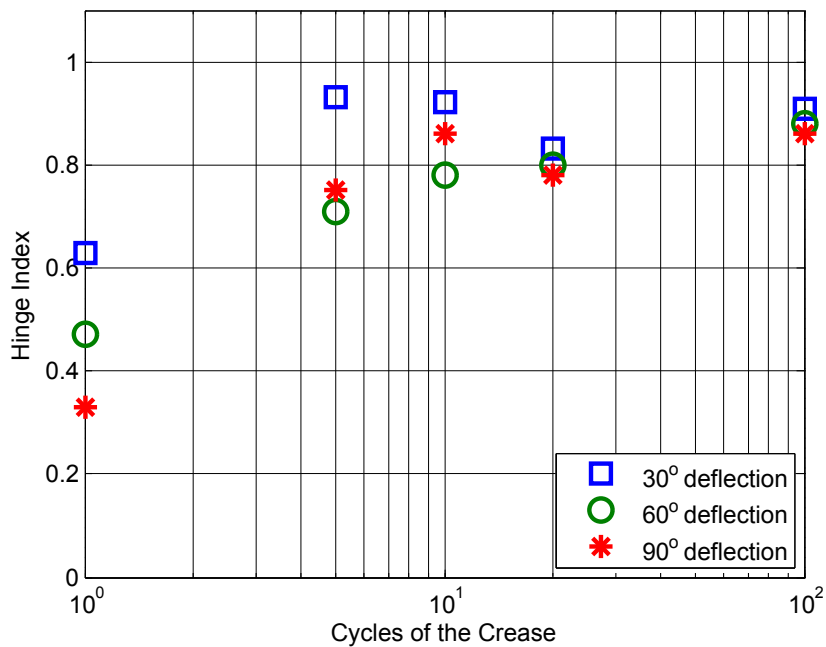

Figure 10. Polypropylene ( $0.25 \mathrm{~mm}$ thick): hinge index verses cycles of the crease.

100 cycles. Some of the samples cracked or failed at the crease well below 100 cycles. The hinge index increased with additional cycles asymptotically, approaching unity. Paper increased its hinge index the most within the first 5 cycles, as shown in Fig. 9; whereas polypropylene continued to increase its hinge index over the 100 cycles, as shown in Fig. 10. ABS shows a distinct positive relationship, as shown in Fig. 11, when plotted on a linear-log plot. Tyvek ${ }^{\circledR}$ exhibits behavior similar to that of paper when cycled, as shown in Fig. 12. Fatigue data for various materials are shown in Table 5, where the cycle to crack was the number of cycles until separation of the material was observed and failure was the number of cycles at fracture or catastrophic crack that resulted in significantly different motion.

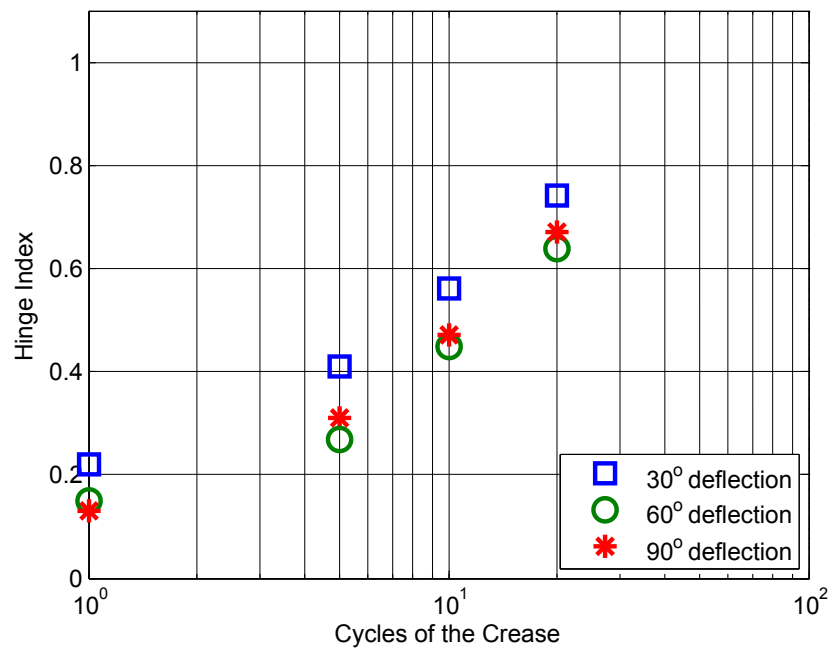

Figure 11. ABS ( $0.25 \mathrm{~mm}$ thick): hinge index verses cycles of the crease. The ABS sample fractured before 100 cycles were reached.

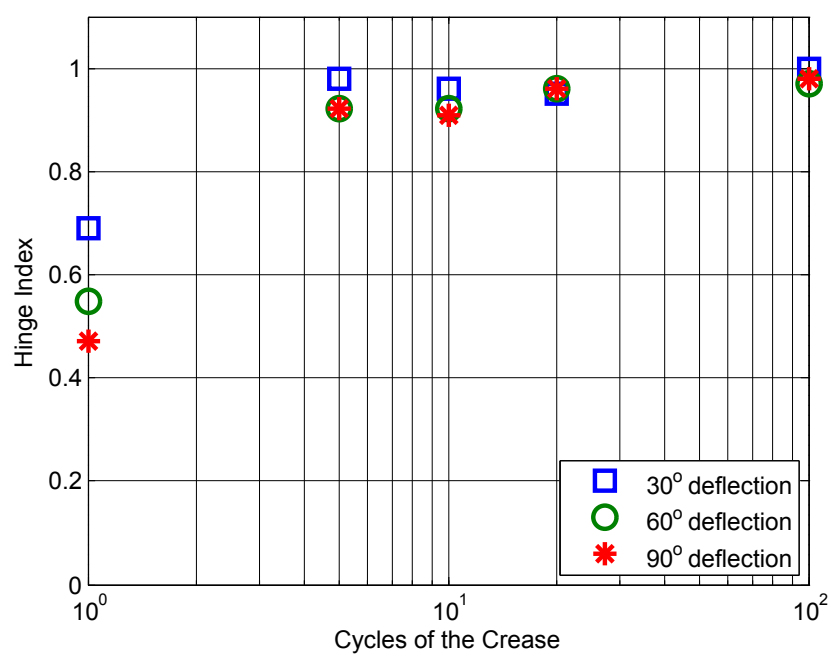

Figure 12. Tyvek $^{\circledR}(0.25 \mathrm{~mm}$ thick): hinge index verses cycles of the crease. A strong asymptotic behavior is seen.

Most fiber-based materials could be cycled extensively before failure. Delamination continued to occur with each cycle; however incremental delamination diminished significantly after 5 cycles. The hinge index approached unity as the number of cycles went to infinity. Fracture/failure of many papers was greater than 100 cycles. Tyvek $^{\circledR}$ likewise delaminated, increased in hinge index with cycles and cycled extensively without failure.

The metal samples failed in the first cycle. Attempts to reverse fold the metal samples resulted in fracture of the sheet. This may be due to strain hardening at the original crease location.

With the polymers, polypropylene gave the highest hingeindex (0.41) after one fold. Polypropylene was also in the subset of polymers that endured more than 100 cycles 
Table 4. Variation in Hinge Indexes and Residual Angles for different thicknesses of Tyvek ${ }^{\circledR}$.

\begin{tabular}{lcccc}
\hline Thickness, mm (in) & $30^{\circ}$ & $60^{\circ}$ & $90^{\circ}$ & $\begin{array}{c}\text { Residual } \\
\text { Angle }^{\circ}\end{array}$ \\
\hline $0.13(0.0050)$ & 0.45 & 0.48 & 0.33 & 9.6 \\
$0.16(0.0063)$ & 0.57 & 0.60 & 0.55 & 4.1 \\
$0.19(0.0075)$ & 0.58 & 0.56 & 0.49 & 0.2 \\
$0.26(0.0103)$ & 0.64 & 0.49 & 0.44 & 0.1 \\
\hline
\end{tabular}

Table 5. Fatigue cycles for $0.25 \mathrm{~mm}$ (0.01 in) thick materials.

\begin{tabular}{|c|c|c|}
\hline Material & Crack & Failure \\
\hline Polycarbonate & 18 & 37 \\
\hline IR Plastic & 3 & 22 \\
\hline Nylon & $>100$ & $>100$ \\
\hline Acetal & 14 & 46 \\
\hline PTFE & $>100$ & $>100$ \\
\hline UHMW Polyethylene & $>100$ & $>100$ \\
\hline Polypropylene & $>100$ & $>100$ \\
\hline Tyvek $^{\circledR}$ & $>100$ & $>100$ \\
\hline Hard Fiber & 2 & 5 \\
\hline Cardstock & $>100$ & $>100$ \\
\hline
\end{tabular}

without experiencing fracture or failure. This is well supported by the extensive use of polypropylene in living hinges.

\subsection{Localized hinge behavior}

Crease locations of each material were examined under a microscope. Fibrous materials demonstrated the effects of delamination, as expected. When the folds were reversed (i.e. the paper was folded back the other direction) the fibers that had been originally pulled apart at the outside edge of the crease (tension) buckled, as shown in Fig. 13.

Metal samples showed yielding of the material at the outside (tension) edge of the crease. Attempts to reverse fold the metal tended to create a new crease near the original crease location. When metal is forced to fold in the same location as the previous fold the crease fractured. This behavior was experienced by all metal samples.

Many of the polymers exhibited a ripple pattern on the compression side and buckled. The polymers yielded on the tension side of the fold. As the plastics were cycled, thinning could be seen at the crease location, as seen in Fig. 14.

Tyvek $^{\circledR}$ is composed of continuous fine polymer fibers, thus it looks similar to the fibrous papers tested. Significant delamination and buckling occur which help create the hinge-like behavior, as seen in Fig. 15.

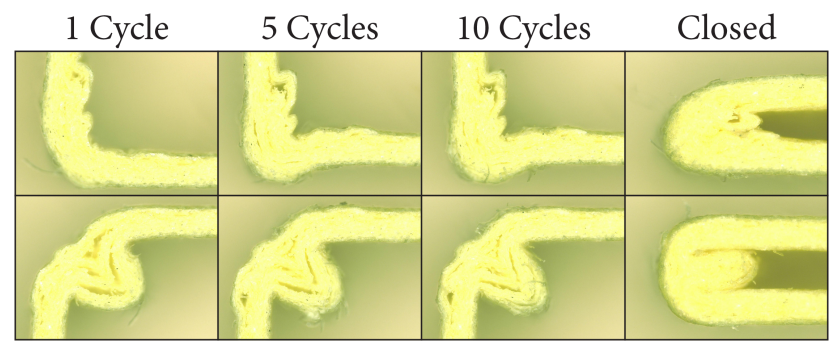

Figure 13. Cyclic fatigue for cardstock. Top row of images are the regular folds, bottom row the reverse folds. Columns from left to right are the following cycles: $1,5,10$, and close fold.

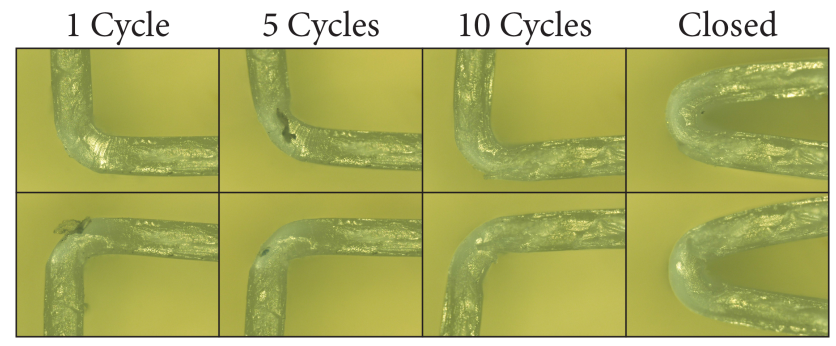

Figure 14. Cyclic behavior for acetal. Delamination is not seen, but localized thinning occurs as tensile stresses on the outside elongate the material.

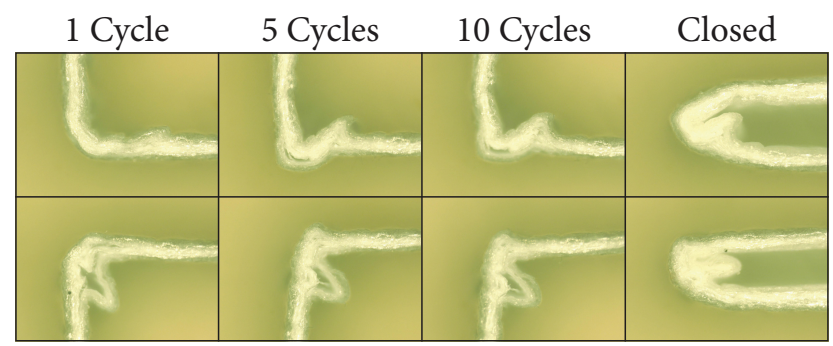

Figure 15. Cyclic behavior for Tyvek ${ }^{\circledR}$. Notice the similarity to the characteristics of the cardstock.

\section{Conclusions}

The results have several implications on direct methods of replicating folding-based designs in non-paper materials. First, the thickness of a material is a significant factor in the hinge index. The thicker the material the higher the hinge index. Second, although the sheet metals have non-zero hinge indexes, they are not well suited for kinetic or reversible folded designs because they do not preference the previous fold. The plastic behavior of folded metals can be used to an advantage for mechanical and shaping situations. Compliant hinge-like behavior in metals may come from alternative methods such as revolute u-form flexures (Ferrell et al., 2011). Third, of the materials tested, polycarbonate offers a low hinge index and returns to its initial shape (low residual angle). Fourth, of the materials tested, Tyvek ${ }^{\circledR}$ comes 
closest to the behavior of paper but with some advantageous physical properties lending it well to situations where paper would quickly degrade. Fifth, unlike paper, Tyvek ${ }^{\circledR}$ has a low residual angle to accompany its high hinge index. Sixth, non-rigid-foldable designs benefit from materials with a low hinge index as the panel warping accommodates for their progression from initial to final states.

An improved characterization of the folding properties of sheet materials provides information that can be used for folded devices. The hinge index was introduced as an approach for quantifying the ability of a material to fold about a crease. Materials with a high index require lower activation forces to traverse their full range of motion, and generally have a higher residual angle while materials with a low index require a higher force for an equivalent range of motion, and have a low residual angle. A better understanding of the hinge index and the cyclic characteristics of the papers, metals and polymers studied provide information to guide designers in the creation of origami-inspired compliant mechanisms. While creasing methods are not the only way to provide such mechanisms, it represents a direct method that may be useful in more straightforward applications. The results of this work will be useful in the design of such compliant mechanisms.

Acknowledgements. This material is based upon work supported by the National Science Foundation and the Air Force Office of Scientific Research under NSF Grant No. 1240417.

Edited by: H. Su

Reviewed by: Y. Klett and one anonymous referee

\section{References}

Allwood, J. M., King, G. P. F., and Duflou, J.: A structured search for application of the incremental sheet forming process by product segmentation, IMechE Part B: Journal of Engineering Manufacture, 219, 239-244, 2005.

Balkcom, D. J. and Mason, M. T.: Robotic origami folding, Int. J. Robot. Res., 27, 613-627, 2008.

Bassik, N., Stern, G. M., and Gracias, D. H.: Microassembly based on hands free origami with bidirectional curvature, Appl. Phys. Lett., 95, 1-3, 2009.

Beex, L. A. A. and Peerlings, R. H. J.: An experimental and compuational study of laminted paperboard creasing and folding, Int. J. Solids Struct., 46, 4192-4207, 2009.

Canford, S., Sen, D., and Buehler, M. J.: Meso-origami: Folding multilayer graphene sheets, Appl. Phys. Lett., 95, 123121, doi:10.1063/1.3223783, 2009.

Dai, J. S.: Stiffness Characteristics of Carton Folds for Packaging, J. Mech. Design, 130, 022305, doi:10.1115/1.2813785, 2008.

Demaine, E. D. and O'Rourke, J.: Geometric Folding Algorithms: Linkages, Origami, Polyhedra, Cambridge University Press, 2007.
Demaine, E. D., Demaine, M. L., Hart, V., Price, G. N., and Tachi, T.: (Non)Existance of Pleated Folds: How Paper Folds Between Creases, Graph. Combinator., 27, 377-397, 2011.

Dureisseix, D.: An Overview of Mechanisms and Patterns with Origami, International Journal of Space Structures, 27, 1-14, 2012.

Elsayed, E. and Basily, B. B.: A Continuous Folding Process for Sheet Materials, Int. J. Mater. Prod. Tech., 21, 217-238, 2004.

Ferrell, D. B., Isaac, Y. F., Magleby, S. P., and Howell, L. L.: Development of Criteria for Lamina Emergent Mechanism Flexures With Specific Applicatoin to Metals, J. Mech. Design, 133, 031009, doi:10.1115/1.4003538, 2011.

Fischer, S., Heimbs, S., Kilchert, S., Klaus, M., and Cluzel, C.: Sandwich Structues with Folded Core: Manufacturing and Mechanical Behavior, SAMPE Europe 30th International Jubilee Conference and Forum, 4B, 256-263, 2009.

Fite, F. B., Ming, N. H., and Ho, T. L.: Foldable Plastic Bowl U.S. patent D567592, 2008.

Gagliardi, D. D. and Gruntfest, I. J.: Creasing and Creaseproofing of Textiles, Text. Res. J., 20, 180-188, 1950.

Gardiner, M.: Oribotics: The Future Unfolds, International Meeting of Origami Science, Mathematics and Education, 5, 127-137, 2011.

Greenberg, H. C., Gong, M. L., Magleby, S. P., and Howell, L. L.: Identifying links between origami and compliant mechanisms, Mech. Sci., 2, 217-225, doi:10.5194/ms-2-217-2011, 2011.

Hearle, J., Grosberg, P., and Backer, S.: Structural Mechanics of Fibers, Yarns and Fabrics, Wiley-Interscience, 1969.

Hochfeld, H.: Method and Machine for Pleating Pliable Materials, US Patent No. 2, 901, 951, 1 September 1959.

Howell, L. L.: Compliant Mechanisms, John Wiley \& Sons, Inc, 2001.

Jacobsen, J. O., Chen, G., Howell, L. L., and Magleby, S. P.: Lamina Emergent Torsional (LET) Joint, Mech. Mach. Theory, 44, 2098 2109, 2009.

Jacobsen, J. O., Winder, B. G., Howell, L. L., and Magleby, S. P.: Lamina Emergent Mechanisms and Their Basic Elements, Journal of Mechanisms and Robotics, 2, 011003 , doi:10.1115/1.4000523, 2010

Karakerezis, A., Doulgeri, Z., and Petridis, V.: A Robotic Gripping System With Consideration of Grasping Flat Non Rigid Materials, Industrial Electronics, Control and Instrumentation, 1994. IECON '94., 20th International Conference on, 2, 936941, 1994.

Kergosien, Y. L., Gotoda, H., and Kunii, T. L.: Bending and Creasing Virtual Paper, IEEE Comput. Graph., January 1994, 40-48, 1994.

Klett, Y. and Drechsler, K.: Designing Technical Tessellations, International Meeting of Origami Science, Mathematics, and Education, 5, 305-322, 2011.

Liu, Y., Boyles, J. K., Genzer, J., and Dickey, M. D.: Self-folding of polymer sheets using local light absorption, The Royal Society of Chemistry, Soft Matter, 8, 1764-1769, 2012.

Marsh, J. T.: Crease Resisting Fabrics, Reinhold Publishing Corporation, 1962.

Martinez, R. V., Fish, C. R., Chen, X., and Whitesides, G. M.: Elastomeric Origami: Programmable Paper-Elastomer Composites as Pneumatic Actuators, Adv. Funct. Mater., 22, 1376-1384, 2012. 
Morton, W. and Hearle, J.: The Textile Institute, Butterworth and Co. LTD, 1962.

Nagasawa, S., Fukuzawa, Y., Yamaguchi, T., Tsukatani, S., and Katayama, I.: Effect of crease depth and crease deviation on folding deformation characteristics of coated paperboard, Journal of Material Processing Technology, 140, 157-162, 2003.

Rothemund, P. W. K.: Folding DNA to create nanoscale shapes and patterns, Nature, 440, 297-302, 2006.

Ryu, J., D'Amato, M., and Cui, X.: Photo-origami-Bending and folding polymers with light, Appl. Phys. Lett., 100, 161908, doi:10.1063/1.3700719, 2012.

Swab, J. J. G.: Expansion Envelope U.S. patent 2188730, 1940.

Tachi, T.: Rigid-Foldable Thick Origami, International Meeting of Origami Science, Mathematics, and Education, 5, 253-263, 2011.
Vigo, T. L.: Textile Processing and Properties: Preparation, Dyeing, Finishing and Performance, Elsevier, 1994.

Wilding, S. E., Howell, L. L., and Magleby, S. P.: Introduction of planar compliant joints designed for combined bending and axial loading conditions in lamina emergent mechanisms, Mech. Mach. Theory, 56, 1-15, 2012.

Willis, A. M.: Collapsible Kayak U.S. patent 8316788B2, 2012.

Wingate, I. B. and Mohler, J. F.: Textile Fabrics and their Selection, Prentice Hall, Inc, 1984.

$\mathrm{Wu}, \mathrm{W}$. and You, Z.: Modeling rigid origami with quaternions and dual quaternions, Proceedings of the Royal Society, 466, 2155$2174,2010$. 